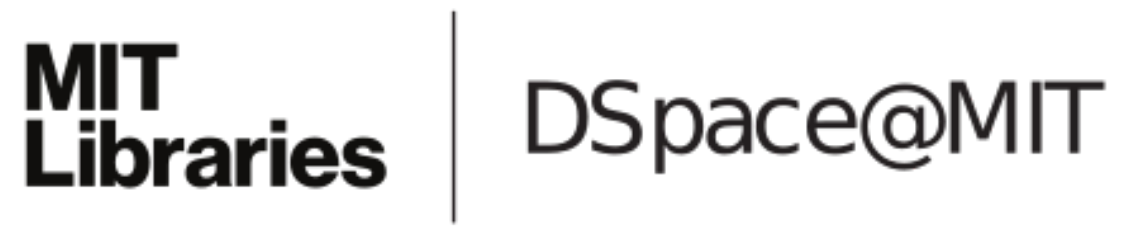

\author{
MIT Open Access Articles
}

Zeroth Poisson homology of symmetric powers of isolated quasihomogeneous surface singularities

The MIT Faculty has made this article openly available. Please share how this access benefits you. Your story matters.

Citation: Eting of, Pavel, and Travis Schedler. "Zeroth Poisson homology of symmetric powers of isolated quasihomogeneous surface singularities." Journal für die reine und angewandte Mathematik (Crelles Journal) 2012, no. 667 (January 2012).

As Published: http://dx.doi.org/10.1515/crelle.2011.124

Publisher: Walter de Gruyter

Persistent URL: http://hdl.handle.net/1721.1/79893

Version: Original manuscript: author's manuscript prior to formal peer review

Terms of use: Creative Commons Attribution-Noncommercial-Share Alike 3.0 


\title{
ZEROTH POISSON HOMOLOGY OF SYMMETRIC POWERS OF ISOLATED QUASIHOMOGENEOUS SURFACE SINGULARITIES
}

\author{
PAVEL ETINGOF AND TRAVIS SCHEDLER
}

\begin{abstract}
Let $X \subset \mathbb{C}^{3}$ be a surface with an isolated singularity at the origin, given by the equation $Q(x, y, z)=0$, where $Q$ is a weighted-homogeneous polynomial. In particular, this includes the Kleinian surfaces $X=\mathbb{C}^{2} / G$ for $G<\mathrm{SL}_{2}(\mathbb{C})$ finite. Let $Y:=S^{n} X$ be the $n$-th symmetric power of $X$. We compute the zeroth Poisson homology $\operatorname{HP}_{0}(Y)$, as a graded vector space with respect to the weight grading. In the Kleinian case, this confirms a conjecture of Alev, that $\mathrm{HP}_{0}\left(\mathbb{C}^{2 n} /\left(G^{n} \rtimes S_{n}\right)\right) \cong \mathrm{HH}_{0}\left(\right.$ Weyl $\left._{2 n}^{G^{n} \rtimes S_{n}}\right)$, where Weyl ${ }_{2 n}$ is the Weyl algebra on $2 n$ generators. That is, the Brylinski spectral sequence degenerates in this case. In the elliptic case, this yields the zeroth Hochschild homology of symmetric powers of the elliptic algebras with three generators modulo their center, $A_{\gamma}$, for all but countably many parameters $\gamma$ in the elliptic curve.
\end{abstract}

\section{Contents}

1. Introduction

1.1. $\quad$ Main result

1.2. Hochschild homology of deformations and Alev's conjecture

1.3. General symmetric products and Poisson-invariant functionals

1.4. A $\mathbb{C}^{*}$-equivariant vector bundle on $\mathbb{P}^{1}$

2. Proof of Theorem 1.4.17

3. Proof of Theorem 1.1.13 when $X$ is not of type $A_{m-1}$

4. Proof of Theorem 1.1.13 in the $A_{m-1}$ case

5. Proof of Theorems 1.2.1 and 1.2.2 and Corollary 1.2 .3

6. Acknowledgements

References

\section{INTRODUCTION}

1.1. Main result. Let $a, b, c$ be positive integers, and equip $\mathbb{C}[x, y, z]$ with a weight grading in which $|x|=a,|y|=b$, and $|z|=c$. In this paper, we are interested in surfaces $X \subset \mathbb{C}^{3}$ with an isolated singularity at the origin, cut out by a polynomial $Q(x, y, z)=0$, which is weighted-homogeneous of degree $d$. Such surfaces were first studied systematically by Saito [Sai87. For convenience, we also assume that $a \leq b \leq c$.

The surface $X$ is equipped with a standard Poisson bracket, given by the bivector

$$
\left.\pi:=\left(\frac{\partial}{\partial x} \wedge \frac{\partial}{\partial y} \wedge \frac{\partial}{\partial z}\right)\right\lrcorner(d Q),
$$

Date: July 9, 2009. 
where $\lrcorner$ is the natural contraction operation, which in this case produces a bivector from a trivector and a one-form. The above bivector is, moreover, weight-homogeneous of degree $\kappa:=d-(a+b+c)$, and is a Poisson bivector (i.e., $\{\pi, \pi\}=0$, where $\{$,$\} is the Schouten-$ Nijenhuis bracket). Hence it produces a Poisson bracket of degree $\kappa$.

In particular, when $\kappa<0, X$ has a Kleinian singularity, i.e., $X \cong \mathbb{C}^{2} / G$ where $G<\mathrm{SL}_{2}(\mathbb{C})$ is a finite subgroup. These finite subgroups have a well-known classification, and up to equivalence, we have:

$$
\begin{gathered}
A_{m-1}: G=\mathbb{Z} / m, a=2, b=c=m, Q=x^{m}+y^{2}+z^{2}, \\
D_{m+2}: G=\widetilde{D_{2 m}}, a=2, b=m, c=m+1, Q=x^{m+1}+x y^{2}+z^{2}, \\
E_{6}: G=\widetilde{A_{4}}, a=3, b=4, c=6, Q=x^{4}+y^{3}+z^{2}, \\
E_{7}: G=\widetilde{S_{4}}, a=4, b=6, c=9, Q=x^{3} y+y^{3}+z^{2}, \\
E_{8}: G=\widetilde{A_{5}}, a=6, b=10, c=15, Q=x^{5}+y^{3}+z^{2} .
\end{gathered}
$$

Here we set the degree $\kappa$ of the bracket to -2 in the $A$ case and -1 for the $D$ and $E$ cases.

The case $\kappa=0$ (i.e., $d=a+b+c$ ) is called the elliptic case, and, up to equivalence, the surface has one of the following forms, for some $\lambda \in \mathbb{C}^{\times}$:

$$
\begin{gathered}
\widetilde{E}_{6}: a=b=c=1, Q=x^{3}+y^{3}+z^{3}+\lambda x y z, \\
\widetilde{E}_{7}: a=b=1, c=2, Q=x^{4}+y^{4}+z^{2}+\lambda x y z, \\
\widetilde{E}_{8}: a=1, b=2, c=3, Q=x^{6}+y^{3}+z^{2}+\lambda x y z .
\end{gathered}
$$

Let $X^{(n)}:=S^{n} X$ be the $n$-th symmetric power of $X$, which is a singular affine Poisson variety. In this paper, we compute explicitly the zeroth Poisson homology of $X^{(n)}$, as a graded vector space using the weight grading. To describe this, recall the Jacobi ring of $X$,

$$
J_{Q}:=\mathbb{C}[x, y, z] /\left(Q_{x}, Q_{y}, Q_{z}\right),
$$

where $Q_{x}, Q_{y}$, and $Q_{z}$ are the partial derivatives of $Q$ with respect to $x, y$, and $z$, respectively. Then, $J_{Q}$ is finite-dimensional, and its dimension is called the Milnor number, and denoted by $\mu_{Q}$.

For any graded vector space $V$ with finite-dimensional graded components, let $h(V ; t)$ denote its Hilbert series.

Recall that, for any Poisson algebra $A$, its zeroth Poisson homology is defined as

$$
\mathrm{HP}_{0}(A):=A /\{A, A\} \text {. }
$$

In AL98, Alev and Lambre showed that

$$
\operatorname{HP}_{0}\left(\mathcal{O}_{X}\right) \cong J_{Q}
$$

as weight-graded vector spaces. Here $\mathcal{O}_{X}$ denotes the global functions on $X$ (which is affine).

It will be convenient to combine the linear duals of the homology groups $\operatorname{HP}_{0}\left(\mathcal{O}_{X^{(n)}}\right)$, for $n \geq 0$, into one bigraded algebra $\bigoplus_{n \geq 0} \operatorname{HP}_{0}\left(\mathcal{O}_{X^{(n)}}\right)^{*}$, with the multiplication given by the symmetrization maps $\mathcal{O}_{X^{(i)}}^{*} \otimes \mathcal{O}_{X^{(j)}}^{*} \rightarrow \mathcal{O}_{X^{(i+j)}}^{*}$. The main result of this paper is 
Theorem 1.1.13. $\bigoplus_{n \geq 0} \mathrm{HP}_{0}\left(\mathcal{O}_{X^{(n)}}\right)^{*}$ is isomorphic, as a bigraded algebra, to a free commutative algebra generated by a bigraded vector space $L$ with Hilbert series

$$
h\left(L ; t^{-1}, s\right)=\frac{h\left(J_{Q} ; t\right) s}{1-t^{d} s} .
$$

Here, the exponent of $t$ is the weight, and the exponent of $s$ is the corresponding symmetric power of $X$. Note that there is a $t^{-1}$ since, by convention, the weights are negated when we take the dual.

We may thus write the Hilbert series of $\bigoplus_{n \geq 0} \mathrm{HP}_{0}\left(\mathcal{O}_{X^{(n)}}\right)$ itself by the following formula. Write $h\left(J_{Q} ; t\right)=t^{n_{1}}+\cdots+t^{n_{r}}$ (in the Kleinian case, the numbers $m_{i}:=n_{i}+1$ (in types $D, E$ ) or $m_{i}:=\frac{n_{i}}{2}+1$ (in type $A$ ) are the Coxeter exponents associated to the corresponding finite Weyl group of type ADE, which has Coxeter number $h=d(\operatorname{types} D, E)$ or $h=\frac{d}{2}$ (type $A)$ ).

$$
h\left(\bigoplus_{n \geq 0} \mathrm{HP}_{0}\left(\mathcal{O}_{X^{(n)}}\right) ; t, s\right)=\prod_{i=1}^{r} \prod_{j \geq 0} \frac{1}{1-t^{n_{i}+j d_{s}} s^{j+1}} .
$$

1.2. Hochschild homology of deformations and Alev's conjecture. In the Kleinian case, i.e., (1.1.2)-(1.1.6),$X \cong \mathbb{C}^{2} / G$ for the listed finite subgroup $G<\mathrm{SL}_{2}(\mathbb{C})$. Using this, one has a canonical quantization of $\mathcal{O}_{X^{(n)}}$, namely Weyl ${ }_{2 n}^{G^{n} \rtimes S_{n}}=$ Sym $^{n}$ Weyl $_{2}^{G}$, where the Weyl algebras are defined as Weyl $_{2}=\mathbb{C}\langle x, y\rangle /([x, y]-1)$ and Weyl $_{2 n}=$ Weyl $_{2}^{\otimes n}$, and $G^{n} \rtimes S_{n}$ is the semidirect product where $S_{n}$ acts by permuting components (i.e., the wreath product of $G$ with $\left.S_{n}\right)$, viewed as a subgroup of $\operatorname{Sp}_{2 n}(\mathbb{C})$.

Here, by "quantization" of a graded Poisson algebra $B$ with bracket of degree $-f$ for some $f>0$, we mean a filtered associative algebra $A=\bigcup_{m} A_{\leq m}$ such that $\operatorname{gr}(A)=B$, and such that, for every $a \in A_{\leq m}, b \in A_{\leq n}$, we have $a b-b a \in A_{\leq(m+n-f)}$ and the image of $a b-b a$ in $\operatorname{gr}_{m+n-f}(A)$ is $\left\{\operatorname{gr}_{m}(a), \operatorname{gr}_{n}(b)\right\}$. (We could alternatively define this as a deformation quantization satisfying a homogeneity property.)

In this situation, there is a standard Brylinski spectral sequence from $\mathrm{HP}_{0}(B)$ to $\mathrm{HH}_{0}(A):=$ $A /[A, A]$. Moreover, we may equip $\mathrm{HH}_{0}(A)$ with the weight filtration from $A$, and it is easy to see that this spectral sequence preserves the grading, in the sense that each page consists of homogeneous differentials. Thus, the spectral sequence converges to $\mathrm{gr} \mathrm{HH}_{0}(A)$.

One may ask whether the Brylinski spectral sequence degenerates. As a consequence of our main theorem, we may deduce the

Theorem 1.2.1. In the case that $B=\mathcal{O}_{X^{(n)}}$ for $X=\mathbb{C}^{2} / G$ a Kleinian singularity listed in (1.1.2)-(1.1.6), and $A=\mathrm{Weyl}_{2 n}^{G^{m} \rtimes S_{m}}$, the Brylinski spectral sequence $\mathrm{HP}_{0}(B) \Rightarrow \mathrm{grHH}_{0}(A)$ degenerates, yielding an isomorphism of graded vector spaces, $\mathrm{HP}_{0}(B) \cong \operatorname{gr} \mathrm{HH}_{0}(A)$.

This confirms a conjecture of J. Alev [But08, Remark 40]. In the case where $G=\mathbb{Z} / 2=$ $\{ \pm \mathrm{Id}\} \subset \mathrm{Sp}_{2}(\mathbb{C})$, this was proved in the case $n=2$ in $\mathrm{AF}$, and for $n=3$ in [But08], where also some preliminary results and conjectures are given towards general $n$ (again for $G=\mathbb{Z} / 2)$.

We remark that Alev also posed a similar conjecture, which replaces $G^{n} \rtimes S_{n}$ as above with finite Weyl groups $W<\mathrm{GL}_{n}(\mathbb{C}) \hookrightarrow \mathrm{Sp}_{2 n}(\mathbb{C})$, where the embedding is given by $A \mapsto$ $\left(\begin{array}{cc}A & 0 \\ 0 & \left(A^{t}\right)^{-1}\end{array}\right)$. The case $(\mathbb{Z} / 2)^{n} \rtimes S_{n}$ then identifies with the Weyl groups of type $B_{n}$ (and 
also with type $C_{n}$ ). We do not know whether this conjecture holds for general Weyl groups of types other than $B$ (or $C$ ), although it was verified in types $D_{2}=A_{1} \times A_{1}$ and $D_{3}=A_{3}$ in [But08].

To deduce Theorem 1.2.1 from Theorem 1.1.13, one uses AFLS00, which gives a general formula for the dimension of $\mathrm{HH}_{0}\left(\mathrm{Weyl}_{2 n}^{G}\right)$ for arbitrary $n$ and $G<\mathrm{Sp}_{2 n}(\mathbb{C})$. However, we will use only the $n=1$ case and a general result from [EO06, §3] to make this more transparent.

In the non-Kleinian cases, the Poisson bracket does not have negative degree, so the above does not apply. However, following [EG07] (e.g., Theorems 3.4.4 and 3.4.5), one may always produce a deformation quantization of $\mathcal{O}_{X}$, i.e, a $\mathbb{C}[[\hbar]]$-algebra $A_{\hbar}$ which is isomorphic to $\mathcal{O}_{X}[[\hbar]]$ as a $\mathbb{C}[[\hbar]]$-module, such that $A_{\hbar} /(\hbar) \cong \mathcal{O}_{X}$ and such that $[a, b]=\hbar\{a, b\}+O\left(\hbar^{2}\right)$ for all $a, b \in \mathcal{O}_{X} \subset \mathcal{O}_{X}[[\hbar]] \cong A_{\hbar}$. For such a deformation quantization, we may similarly deduce the

Theorem 1.2.2. The Brylinski spectral sequence $\operatorname{HP}_{0}\left(\mathcal{O}_{X^{(n)}}\right)((\hbar)) \Rightarrow \operatorname{grHH} H_{0}\left(\operatorname{Sym}^{n} A_{\hbar}\left[\hbar^{-1}\right]\right)$ degenerates.

This generalizes the theorem above.

In the case that the Poisson bracket has degree zero, there exist not merely formal but actual, homogeneous quantizations of $A$, the Artin-Tate-Odesski-Sklyanin-type algebras $A_{\gamma}$ modulo their center (e.g., ATdB90, Ste97]; see also [EG07, §3.5]). Here the parameter $\hbar$ is replaced by a point $\gamma$ on an elliptic curve. For such algebras, we deduce

Corollary 1.2.3. For all but countably many parameters $\gamma$, we have a noncanonical isomorphism of weight-graded vector spaces, $\mathrm{HH}_{0}\left(\operatorname{Sym}^{n} A_{\gamma}\right) \cong \mathrm{HP}_{0}\left(\mathcal{O}_{X^{(n)}}\right)$, and moreover,

$$
\bigoplus_{n} \mathrm{HH}_{0}\left(\operatorname{Sym}^{n} A_{\gamma}\right)^{*} \cong \operatorname{Sym}\left(\mathrm{HH}_{0}\left(A_{\gamma}\right)^{*}[t]\right) \text {, }
$$

as bigraded algebras (noncanonically), where $\mathrm{HH}_{0}\left(\operatorname{Sym}^{n} A_{\gamma}\right)^{*}$ has degree $n$, and $t$ has degree 1 and weight $-d !$

Note that, when $\gamma$ is a point of finite order of the elliptic curve, then $\mathrm{HH}_{0}\left(A_{\gamma}\right)$ is infinitedimensional, and the isomorphism $\mathrm{HH}_{0}\left(A_{\gamma}\right) \cong \mathrm{HP}_{0}\left(\mathcal{O}_{X}\right)$ fails. We expect that these are exactly the countably many $\gamma$ mentioned in the corollary.

Moreover, we deduce the same result for the filtered deformations of these elliptic algebras as in [VdB01, EG07]:

Corollary 1.2.5. For all but countably many $\gamma$, if $A_{\gamma}^{\prime}$ is an associative algebra with an ascending filtration such that $\operatorname{gr} A_{\gamma}^{\prime}=A_{\gamma}$, then $\operatorname{grHH}_{0}\left(\operatorname{Sym}^{n} A_{\gamma}^{\prime}\right) \cong \mathrm{HH}_{0}\left(\operatorname{Sym}^{n} A_{\gamma}\right)$ as graded vector spaces.

Remark 1.2.6. The isomorphisms above don't have anything to do with the specific quantizations. Generally, the $n$-th symmetric power of any formal or generic filtered (or graded) quantization $A^{\prime}$ of $\mathcal{O}_{X}$ has $\mathrm{HH}_{0}\left(\operatorname{Sym}^{n} A^{\prime}\right) \cong \mathrm{HP}_{0}\left(\operatorname{Sym}^{n} \mathcal{O}_{X}\right) \otimes K$, as filtered (or graded) vector spaces, where $K$ is the base field for the deformation (i.e., $K=\mathbb{C}((\hbar))$ for a formal deformation, and $K=\mathbb{C}$ for a generic enough point of an actual deformation). Similarly, for any formal or generic filtered/graded Poisson deformation $B^{\prime}$ of $\mathcal{O}_{X}$, we have

\footnotetext{
${ }^{1}$ In general, there is a canonical map from the RHS to the LHS as degree-graded algebras, but it is not an isomorphism, nor a map of weight-graded algebras; see Remark 5.0 .78
} 
$\mathrm{HP}_{0}\left(\mathrm{Sym}^{n} B^{\prime}\right) \cong \mathrm{HP}_{0}\left(\mathrm{Sym}^{n} \mathcal{O}_{X}\right) \otimes K$, as filtered/graded vector spaces. Note that the semiuniversal formal deformations were classified in [EG07], and the actual elliptic deformations were deduced as well (these were studied earlier by a different method in [VdB01]).

1.3. General symmetric products and Poisson-invariant functionals. It is convenient to replace Poisson homology with invariant functionals, as follows. Let $X$ be an affine Poisson variety. Let $\mathfrak{g}_{X} \subseteq \Gamma(X, T X)$ be the Lie algebra of Hamiltonian vector fields, which is the same as $\mathcal{O}_{X} / Z\left(\mathcal{O}_{X}\right)$, viewing $\mathcal{O}_{X}$ as a Lie algebra, and denoting by $Z\left(\mathcal{O}_{X}\right)$ the Poisson center of $\mathcal{O}_{X}$. As is standard, invariants of a Lie algebra action of $\mathfrak{g}$ on $A$ are denoted by $A^{\mathfrak{g}}:=\{a \in A: g(a)=0, \forall g \in \mathfrak{g}\}$.

We now reformulate the problem of computing Poisson homology of the varieties $X^{(n)}$ :

Proposition 1.3.1. For every affine Poisson variety $X$, there is a canonical graded algebra isomorphism

$$
\bigoplus_{n \geq 0} \mathrm{HP}_{0}\left(X^{(n)}\right)^{*} \cong \mathbb{C}\left[\mathcal{O}_{X}\right]^{\mathfrak{g} X}=\bigoplus_{n \geq 0}\left(\operatorname{Sym}^{n}\left(\mathcal{O}_{X}\right)^{*}\right)^{\mathfrak{g}_{X}}
$$

Here, $\mathbb{C}\left[\mathcal{O}_{X}\right]$ denotes the polynomial functions on the (infinite-dimensional) vector space $\mathcal{O}_{X}$, which is equipped with the coadjoint action of $\mathfrak{g}_{X}$. The proof is easy and short:

Proof. We have $H P_{0}\left(X^{(n)}\right)=\left(\operatorname{Sym}^{n} \mathcal{O}_{X}\right) /\left\{\operatorname{Sym}^{n} \mathcal{O}_{X}, \operatorname{Sym}^{n} \mathcal{O}_{X}\right\}$. We claim that there is an identification

$$
\left\{\operatorname{Sym}^{n} \mathcal{O}_{X}, \operatorname{Sym}^{n} \mathcal{O}_{X}\right\}=\mathfrak{g}_{X}\left(\operatorname{Sym}^{n} \mathcal{O}_{X}\right) .
$$

Note that we have an obvious inclusion of vector spaces, $\mathcal{O}_{X} \subseteq$ Sym $^{n} \mathcal{O}_{X}$, given by $f \mapsto$ $f \& 1 \& \cdots \& 1$. Using this, the inclusion $\supseteq$ above follows from the equality

$$
g\left(f_{1} \& f_{2} \& \cdots \& f_{n}\right)=\left\{g, f_{1} \& \cdots \& f_{n}\right\} .
$$

For the inclusion $\subseteq$, we use the fact that $\operatorname{Sym}^{n} \mathcal{O}_{X}$ is generated, as a commutative algebra, by the subspace $\mathcal{O}_{X} \subseteq \operatorname{Sym}^{n} \mathcal{O}_{X}$ : this follows inductively on $n$ by an easy argument. Then, we use the fact that, for any Poisson algebra $A$ which is generated as a commutative algebra 2 by $V \subseteq A$, we have $\{A, A\}=\{V, A\}$, by the identity

$$
\{a b, c\}=\{a, b c\}+\{b, c a\} .
$$

1.4. A $\mathbb{C}^{*}$-equivariant vector bundle on $\mathbb{P}^{1}$. We return to the surface $X=Z(Q)$ from \$1.1. In the Kleinian $A_{m-1}$-case, we will give a short and self-contained proof of Theorem 1.1 .13 in $₫ 4$ (which would deserve mention even if the proof below extended to this case).

The main step of the proof for all other cases is to write $\mathbb{C}\left[\mathcal{O}_{X}\right]^{\mathfrak{g}_{X}}$ as the algebra of global sections of a certain infinite-dimensional $\mathbb{C}^{*}$-equivariant vector bundle on $\mathbb{P}^{1}$, whose definition and structure we explicitly describe in this section.

Henceforth, we assume that we are not in the Kleinian type $A_{m-1}$ case. This has the following important consequence:

Lemma 1.4.1. Suppose that $X$ is not of Kleinian type $A_{m-1}$. Then, all nonzero homogeneous Hamiltonian vector fields $\xi_{f}$ have positive degree. In particular, $\langle x, y\rangle \cap\left\{\mathcal{O}_{X}, \mathcal{O}_{X}\right\}=0$.

\footnotetext{
${ }^{2}$ In fact, $\{A, A\}=\{V, A\}$ assuming only that $A$ is generated as a Poisson algebra by $V$, using (1.3.5) and the Jacobi identity.
} 
Proof. In the non-Kleinian case, it is clear that $\xi_{f}$ has positive degree for all noncentral $f$, since the Poisson bracket has nonnegative degree and $\mathbb{C}$ is central. In the Kleinian case, looking at (1.1.2)-(1.1.6), only in the type $A_{m-1}$ case is there a Hamiltonian vector field of nonpositive degree (in particular, $\xi_{x}$ has zero degree, and in type $A_{1}$, also $\xi_{y}$ and $\xi_{z}$ ). Then, since $a \leq b \leq c$, for all homogeneous $f, g$ with $\{f, g\} \neq 0$, we have $|\{f, g\}| \geq\left|\xi_{x}(y)\right|>b$, which implies the final statement.

Next, for any $m \geq 0$, let $\left(\mathcal{O}_{X}\right)_{m}$ denote the subspace of $\mathcal{O}_{X}$ of weighted degree $m$. It is convenient to consider, rather than $\mathbb{C}\left[\mathcal{O}_{X}\right]$, the subalgebra

$$
\mathcal{F}(X):=\operatorname{Sym}\left(\bigoplus_{m \geq 0}\left(\left(\mathcal{O}_{X}\right)_{m}\right)^{*}\right)
$$

The entire algebra $\mathbb{C}\left[\mathcal{O}_{X}\right]$ is the completion of $\mathcal{F}(X)$ by the weight grading. In other words, $\mathcal{F}(X)$ is the algebra of continuous polynomial functions on the completion $\mathcal{O}_{\widehat{X}}=$ $\mathbb{C}[[x, y, z]] /(Q)$ with respect to the weight grading. We may view $\mathcal{O}_{\widehat{X}}$ as a pro-scheme (with limit taken over finite-dimensional affine spaces), and in this sense, $\mathcal{F}(X)=\mathbb{C}\left[\mathcal{O}_{\widehat{X}}\right]$. Note that $\mathbb{C}\left[\mathcal{O}_{X}\right]^{\mathfrak{g}_{X}}$ is also a completion of $\mathcal{F}(X)^{\mathfrak{g}_{X}}$. In our case, in fact, $\left(\operatorname{Sym}^{n}\left(\mathcal{O}_{X}\right)^{*}\right)^{\mathfrak{g}_{X}}$ will turn out to be finite-dimensional for each $n$, and hence $\mathbb{C}\left[\mathcal{O}_{X}\right]^{\mathfrak{g}_{X}}=\mathbb{C}\left[\mathcal{O}_{\widehat{X}}\right]^{\mathfrak{g}_{X}}$.

Let $V:=\langle x, y\rangle \subset \mathcal{O}_{\widehat{X}}$. Fix a graded complement $\mathcal{O}_{\widehat{X}}^{0}$ to $V$ containing all Poisson brackets. Thus, $\mathcal{O}_{\widehat{X}}=V \times \mathcal{O}_{\widehat{X}}^{0}$. Note that functionals in $\mathcal{F}(X)$ are the same as regular functions on $(V \backslash\{0\}) \times \mathcal{O}_{\widehat{X}}^{0}$.

Lemma 1.4.3. The invariants $\mathcal{F}(X)^{\mathfrak{g}_{X}}$ can be noncanonically identified with regular functions on the total space of the pro-vector bundle $Y^{\prime}$ on $V \backslash\{0\}$ with fiber over $(\alpha, \beta)$ given by

$$
Y_{(\alpha, \beta)}^{\prime}=\mathcal{O}_{\widehat{X}}^{0} /\left\{\alpha x+\beta y, \mathcal{O}_{\widehat{X}}\right\} \text {. }
$$

The lemma will be proved in Section 3. Let us explain why (1.4.4) indeed defines a provector bundle. Note that $Y^{\prime}$ is a pro-coherent sheaf which is pulled back from $\mathbb{P}^{1}$. Next, viewing $\mathcal{O}_{\widehat{X}}$ as a constant pro-vector bundle, we may view $Y^{\prime}$ as the cokernel of the procoherent sheaf map,

$$
\mathcal{O}_{\widehat{X}} \otimes \mathcal{O}(-1) \rightarrow \mathcal{O}_{\widehat{X}}^{0}, \quad f \otimes(\alpha x+\beta y) \mapsto\{f, \alpha x+\beta y\}
$$

This map descends to

$$
\mathcal{O}_{\widehat{X}} / \mathbb{C}[[\alpha x+\beta y]] \otimes \mathcal{O}(-1) \rightarrow \mathcal{O}_{\widehat{X}}^{0},
$$

where $\mathcal{O}_{\widehat{X}} / \mathbb{C}[[\alpha x+\beta y]]$ is the quotient of $\mathcal{O}_{\widehat{X}}$ by the sub-pro-vector bundle $\mathbb{C}[[\alpha x+\beta y]] \cong$ $\prod_{i \geq 0} \mathcal{O}(-1)^{\otimes i}$.

We claim that (1.4.6) is injective on fibers. This follows by computing that $\mathbb{C}[[\alpha x+\beta y]]$ is the kernel of $\{\alpha x+\beta y,-\}$, see Lemma 2.0.19. Hence, this is a pro-vector bundle map, and the cokernel, $Y^{\prime}$, is indeed a pro-vector bundle.

Next, note that $Y^{\prime}$ is equipped with a $\mathbb{C}^{*}$-equivariant structure with respect to the $\mathbb{C}^{*}$ action on $V$, given by, for $w \in \mathbb{C}^{*}$,

$$
x \mapsto w^{a} x, \quad y \mapsto w^{b} y .
$$

The action on coordinate functions then has the form

$$
\alpha \mapsto w^{-a} \alpha, \quad \beta \mapsto w^{-b} \beta .
$$


Furthermore, $Y^{\prime}$ is pulled back from a pro-vector bundle $Y$ on $\mathbb{P}^{1}$. We may thus regard $\mathcal{F}(X)^{\mathfrak{g}_{X}}$ as the regular functions on the total space of the pro-vector bundle

$$
E:=Y \oplus \mathcal{O}(-1)
$$

on $\mathbb{P}^{1}$. These pro-bundles are also $\mathbb{C}^{*}$-equivariant.

Note that representations $W$ of $\mathbb{C}^{*}$ may also be viewed as graded vector spaces, with action of $w$ in degree $m$ by multiplication by $w^{m}$. Thus, we will use the notation $h(W ; t)$ for the character of $W$ viewed as a representation of $\mathbb{C}^{*}$, i.e., the Hilbert series of $W$ where $W$ is viewed as a graded vector space (rather than a vector space with $\mathbb{C}^{*}$-action).

Next, we describe the structure of $Y$, which will imply the main theorem. First, recall the following basic facts about $\mathbb{C}^{*}$-equivariant vector bundles on $\mathbb{P}^{1}$ :

Definition 1.4.10. Let $\mathcal{O}(n)_{m}$ denote $\mathcal{O}(n)$ with the $\mathbb{C}^{*}$-equivariant structure given by the action of $w \in \mathbb{C}^{*}$ on the fiber over $(1,0)$ as multiplication by $w^{m}$.

In particular, the tautological line bundle $\mathcal{O}(-1)$ (which appeared in (1.4.9)) is the equivariant bundle $\mathcal{O}(-1)_{a}$. We will need the following well-known result, whose proof is easy and omitted:

Theorem 1.4.11. Let $\mathbb{P}^{1}$ be equipped with the above $\mathbb{C}^{*}$-action.

(i) Up to isomorphism, any $\mathbb{C}^{*}$-equivariant vector bundle on $\mathbb{P}^{1}$ has a unique decomposition as a sum of line bundles of the form $\mathcal{O}(n)_{m}$.

(ii) For $n \geq 0$,

$$
h\left(\Gamma\left(\mathbb{P}^{1}, \mathcal{O}(n)_{m}\right) ; t\right)=t^{m}\left(1+t^{a-b}+\cdots+t^{n(a-b)}\right) .
$$

Remark 1.4.13. In fact, we will work also with pro- $\mathbb{C}^{*}$-equivariant vector bundles, but only those for which the weight- $m$ subspaces of the fibers at $(0,1)$ and $(1,0)$ are finite-dimensional for all $m \in \mathbb{Z}$. In this case, the above theorem still applies, except that now the pro-bundles will be a direct product of possibly infinitely many $\mathcal{O}(n)_{m}$ (but only finitely many for each value of $m$ ). In particular, the Hilbert series of global sections makes sense.

We may therefore make the following definition:

Definition 1.4.14. For any $\mathbb{C}^{*}$-equivariant vector bundle $U$ on $\mathbb{P}^{1}$ of the form $U \cong \bigoplus_{i} \mathcal{O}\left(p_{i}\right)_{q_{i}}$, write

$$
\chi_{\mathbb{C}^{*}}(U)=\sum_{i} s^{p_{i}} t^{q_{i}}
$$

We extend this notation in the obvious way to pro- $\mathbb{C}^{*}$-equivariant vector bundles whose fibers over $(0,1)$ and $(1,0)$ have finite-dimensional weight- $m$ subspaces for all $m \in \mathbb{Z}$. Now, we may state the main technical result of the paper, which implies Theorem 1.1.13. It will be convenient to use the pro-bundle

$$
\widetilde{Y}:=Y \oplus \mathcal{O}(0)_{a} \oplus \mathcal{O}(0)_{b}
$$

Theorem 1.4.17. We have

$$
\chi_{\mathbb{C}^{*}}(\tilde{Y})=\frac{\left(1-t^{d-a}\right)\left(1-t^{d-b}\right)\left(1-t^{d-c}\right)}{\left(1-t^{a}\right)\left(1-t^{b}\right)\left(1-t^{c}\right)\left(1-t^{d-a} s\right)}
$$

The next section is devoted to the proof of this theorem. 


\section{Proof of Theorem 1.4.17}

The following lemma will be a cornerstone of the proof:

Lemma 2.0.19. The kernel of $\{\alpha x+\beta y,-\}: \mathcal{O}_{\widehat{X}} \rightarrow \mathcal{O}_{\widehat{X}}$ is $\mathbb{C}[[\alpha x+\beta y]] \subset \mathcal{O}_{\widehat{X}}$.

(Note that the inclusion $\mathbb{C}[[\alpha x+\beta y]] \subset \mathcal{O}_{\widehat{X}}$ makes sense since, e.g., $Q \notin \mathbb{C}[[x, y]]$ ).

Proof. We first claim that it is sufficient to consider the case where either $\alpha=0$ or $\beta=0$. First, if $a=b$ (i.e., $|x|=|y|$ ), then we may change bases to replace $\alpha x+\beta y$ with $x$. If $a<b$, then, letting $Z_{f}$ denote the Poisson centralizer of $f$, we have $\operatorname{gr} Z_{\alpha x+\beta y} \subseteq Z_{x}$ when $\alpha \neq 0$. Since $\mathbb{C}[[\alpha x+\beta y]] \subseteq Z_{\alpha x+\beta y}$, when $\alpha \neq 0$ it is sufficient to assume that $\beta=0$.

Suppose $\beta=0$. Let $f \in \mathcal{O}_{\hat{X}}$ be such that $\{x, f\}=0$. We need to show that $f$ is a power series in $x$. Clearly, one may assume without loss of generality that $f$ is a polynomial. Because $X$ is generically symplectic, $f$ must be algebraically dependent on $x$. But it is easy to show that $\mathbb{C}[x]$ is algebraically closed in $\mathbb{C}[x, y, z] /(Q)$ (e.g., any homogeneous element in the algebraic closure of $\mathbb{C}[x]$ would have to be a rational power of $x$, and only nonnegative integer powers of $x$ occur in $\mathbb{C}[x, y, z] /(Q))$, so $f \in \mathbb{C}[x]$. The case where $\alpha=0$ is similar.

Now, in view of Theorem 1.4.11, to prove Theorem 1.4 .17 it suffices to compute the character (Hilbert series) of the vector spaces

$$
V_{q}:=\Gamma\left(\mathbb{P}^{1}, \tilde{Y} \otimes \mathcal{O}(-q)_{0}\right) .
$$

Since $\widetilde{Y}$ is a quotient of a trivial pro-bundle, it suffices to take $q \geq 0$. We obtain the following, which, together with Theorem 1.4.11.(ii), immediately implies Theorem 1.4.17;

Proposition 2.0.21. For $q \geq 0$,

$$
h\left(V_{q} ; t\right)=\frac{t^{q(d-a)}\left(1-t^{d-c}\right)}{\left(1-t^{a}\right)\left(1-t^{b}\right)\left(1-t^{c}\right)} .
$$

Proof. We may identify $V_{q}$ with the space of global sections of $\widetilde{Y}$ which vanish to order $q$ at $(0,1)$, using the injections of sheaves, $\mathcal{O}(n)_{m} \otimes \mathcal{O}(-1)_{0} \cong \mathcal{O}(n-1)_{m} \hookrightarrow \mathcal{O}(n)_{m}$, which, on global sections, are the inclusions of sections vanishing at $(0,1) 3^{3}$

To prove the proposition, we first rewrite the condition of vanishing to order $q$ at $(0,1)$, by explicitly describing the subspace $\left\{\alpha x+\beta y, \mathcal{O}_{\widehat{X}}\right\}$. As in the introduction, let $f_{x}, f_{y}, f_{z}$ denote the partial derivatives of $f \in \mathbb{C}[[x, y, z]]$ with respect to $x, y$, and $z$. We have $\{x, y\}=Q_{z}$, $\{y, z\}=Q_{x}$, and $\{z, x\}=Q_{y}$. Thus,

$$
\{\alpha x+\beta y, f\}=\alpha\left(Q_{z} f_{y}-Q_{y} f_{z}\right)-\beta\left(Q_{z} f_{x}-Q_{x} f_{z}\right) .
$$

In particular, we deduce that

$$
\{\alpha x+\beta y, \mathbb{C}[[x, y]]\}=\mathbb{C}[[x, y]] Q_{z} .
$$

We may use this to compute the global sections of $\widetilde{Y}$. Let $T$ be a graded complement to $\mathbb{C}[[x, y]]$ in $\mathcal{O}_{\widehat{X}}$, so that $\mathcal{O}_{\widehat{X}}=\mathbb{C}[[x, y]] \oplus T$. By Lemma 2.0.19, the kernel of $\{\alpha x+\beta y,-\}$ lies in $\mathbb{C}[[x, y]]$. Hence, we have an exact sequence

$$
0 \rightarrow \mathcal{O}(-1)_{a} \otimes T \rightarrow \mathcal{O}_{\bar{X}}^{0} / \mathbb{C}[[x, y]] Q_{z} \rightarrow Y \rightarrow 0,
$$

\footnotetext{
${ }^{3}$ The map to sections vanishing at $(1,0)$ comes from $\mathcal{O}(n-1)_{m+a-b} \hookrightarrow \mathcal{O}(n)_{m}$, so we could have instead used sections vanishing to order $q$ at $(0,1)$, but with weights shifted by $q(a-b)$. The argument goes through in the same way, swapping $x$ with $y$ and $a$ with $b$.
} 
and since $Y$ is torsion-free, we conclude by taking global sections that

$$
\Gamma\left(\mathbb{P}^{1}, Y\right) \cong \mathcal{O}_{\widehat{X}}^{0} / \mathbb{C}[[x, y]] Q_{z} .
$$

To describe $V_{q}$ for $q>0$, it will be convenient to sometimes work in the larger ring $\mathbb{C}[[x, y, z]]\left[Q_{z}^{-1}\right]$, and define the operators

$$
D_{x}:=\partial_{x}-\frac{Q_{x}}{Q_{z}} \partial_{z}, \quad D_{y}:=\partial_{y}-\frac{Q_{y}}{Q_{z}} \partial_{z} .
$$

These operators make sense on $\mathbb{C}[[x, y, z]]\left[Q_{z}^{-1}\right] /(Q)$ since $D_{x}=-\frac{1}{Q_{z}}$ ad $y$ and $D_{y}=\frac{1}{Q_{z}}$ ad $x$ (or because $D_{x}(Q)=0=D_{y}(Q)$ ). Moreover, it is clear that, if we think of $z$ as implicitly dependent on $x$ and $y$ via $Q=0$, then $D_{x}$ and $D_{y}$ are the derivatives with respect to $x$ and $y$. In particular, on $\mathbb{C}[[x, y]], D_{x}$ and $D_{y}$ restrict to the usual derivative with respect to $x$ and $y$. Finally, we have

$$
\left[D_{x}, D_{y}\right]=0
$$

which follows from the above (one may also directly compute that $\left[D_{x}, D_{y}\right](z)=0$ ).

It now follows from (2.0.23), (2.0.26), and (2.0.27) that $V_{q}$ is identified with the solutions $G \in \mathcal{O}_{\widehat{X}}$ (modulo the subspace $\mathbb{C}[[x, y]] Q_{z}$ ) of the equations

$$
\begin{aligned}
& \exists F_{1}, \ldots, F_{q} \in \mathbb{C}[[x, y, z]] /(Q) \text { s.t. } G=Q_{z} D_{x} F_{1}, \\
& \qquad D_{y} F_{1}=D_{x} F_{2}, \ldots, D_{y} F_{q-1}=D_{x} F_{q} .
\end{aligned}
$$

We break up most of the rest of the proof into lemmas. It suffices to consider homogeneous solutions to the above equations, which we do from now on. In particular, this means we can (and will) work in the uncompleted rings $\mathbb{C}[x, y, z], \mathbb{C}[x, y, z] /(Q), \mathbb{C}[x, y, z]\left[Q_{z}^{-1}\right] /(Q)$, etc.

Lemma 2.0.30. Every homogeneous solution to (2.0.29) has the form

$$
F_{i}=D_{y}^{i-1} D_{x}^{q-i} H, \quad G=Q_{z} D_{x}^{q} H
$$

for some homogeneous element $H \in \mathbb{C}[x, y, z] /(Q)$, which is uniquely determined by the $F_{i}$.

The proof mimics a standard proof of the Poincaré lemma.

Proof. We show, inductively on $j$, that there exist unique homogeneous $H_{i, j}$, for $i+j \leq q$ and $i \geq 1, j \geq 0$, such that (for $j \geq 1$ )

$$
D_{x} H_{i, j}=H_{i, j-1}, \quad D_{y} H_{i, j}=H_{i+1, j-1}, \quad H_{i, 0}:=F_{i} .
$$

Then, it follows that $H=H_{1, q-1}$ has the desired property.

To do this, we use the formula, valid for all homogeneous $f$ of positive degree:

$$
|f| f=a x D_{x} f+b y D_{y} f .
$$

Thus, given any $g$ and $h$, there exists $f$ such that $D_{x} f=g$ and $D_{y} f=h$ if and only if $D_{y} g=D_{x} h$, and in this case, $|f| f=a x g+a y h$. The inductive step therefore follows by setting $g=H_{i, j}$ and $h=H_{i+1, j}$.

Next, we have to find what possible $H$ can arise. This is answered by

Lemma 2.0.34. Let $H \in \mathbb{C}[x, y, z]$ be homogeneous. Then, the following are equivalent:

(i) For all polynomials $f$ of degree $\leq n$, we have

$$
f\left(D_{x}, D_{y}\right) H \in \mathbb{C}[x, y, z]+Q \cdot \mathbb{C}[x, y, z]\left[Q_{z}^{-1}\right] .
$$


(ii) We have

$$
H_{z} \in\left(Q^{n}\right)+(Q)_{z}
$$

Here and below, $(Q)_{z}$ is the partial derivative of the ideal $(Q) \subset \mathbb{C}[x, y, z]$, not the element. As a consequence of the lemma, we deduce that the possible $H$ in (2.0.31) are exactly those satisfying (2.0.36) for $n=q-1$.

Proof. The implication (ii) $\Rightarrow(\mathrm{i})$ is easy: since $D_{x}, D_{y}$ are well-defined on $\mathbb{C}[[x, y, z]]\left[Q_{z}^{-1}\right] /(Q)$, given (2.0.36), we may assume that $Q^{n} \mid H$, and (2.0.35) follows immediately.

Next, we prove (i) $\Rightarrow$ (ii) inductively on $n$. For $n=0$ the assertion is vacuous. Since the assertion does not depend on the choice of $H$ modulo $(Q)$, we may assume inductively that $Q^{n-1} \mid H_{z}$ and (2.0.35) holds. We must prove that, up to adding an element of $(Q)$ to $H$, we have $Q^{n} \mid H_{z}$.

Letting $f\left(D_{x}, D_{y}\right)=D_{x}$ in (2.0.35), we have

$$
H_{x}-\frac{Q_{x}}{Q_{z}} H_{z} \in \mathbb{C}[x, y, z]+Q \cdot \mathbb{C}[x, y, z]\left[Q_{z}^{-1}\right] .
$$

It follows that

$$
Q_{x} H_{z} \in Q_{z} \mathbb{C}[x, y, z]+Q \cdot \mathbb{C}[x, y, z]\left[Q_{z}^{-1}\right],
$$

but since also $H_{z} \in \mathbb{C}[x, y, z]$, we in fact have

$$
Q_{x} H_{z} \in\left(Q, Q_{z}\right) .
$$

Similarly, using $f\left(D_{x}, D_{y}\right)=D_{y}$ in (2.0.35), we have

$$
Q_{y} H_{z} \in\left(Q, Q_{z}\right) \text {. }
$$

By the following Lemma 2.0.50, $H_{z} \in\left(Q, Q_{z}\right)$ itself. This proves (2.0.36) in the case $n=1$.

We proceed now under the assumption that $n \geq 2$. Write $H_{z}=Q^{n-1} h$. Then

$$
D_{x} H-H_{x} \in Q \cdot \mathbb{C}[x, y, z]\left[Q_{z}^{-1}\right] .
$$

As a consequence, (2.0.35) implies that

$$
f\left(D_{x}, D_{y}\right) H_{x} \in \mathbb{C}[x, y, z]+Q \mathbb{C}[x, y, z]\left[Q_{z}^{-1}\right],
$$

for all polynomials $f$ of degree $\leq n-1$. By the inductive hypothesis applied to $H_{x}$, we conclude that

$$
H_{x z} \in\left(Q^{n-1}\right)+(Q)_{z} .
$$

Substituting $H_{z}=Q^{n-1} h$, we find that

$$
(n-1) Q^{n-2} Q_{x} h \in\left(Q^{n-1}\right)+(Q)_{z} .
$$

Next, note that

$$
(Q)_{z} \cap\left(Q^{n-2}\right)=\left(Q^{n-1}\right)_{z}:=\left\{g_{z} \mid g \in\left(Q^{n-1}\right)\right\},
$$

since if $Q \nmid g$, we have $\partial_{z}\left(Q^{j} g\right)=j Q^{j-1} Q_{z} g+Q^{j} g_{z}$, which is in $\left(Q^{j-1}\right)$ but not $\left(Q^{j}\right)$. Applying this to (2.0.44), we deduce that

$$
Q^{n-2} Q_{x} h \in\left(Q^{n-1}\right)+\left(Q^{n-1}\right)_{z}=Q^{n-2}\left(Q, Q_{z}\right) .
$$

Dividing by $Q^{n-2}$, we get that

$$
\begin{gathered}
Q_{x} h \in\left(Q, Q_{z}\right), \\
10
\end{gathered}
$$


and similarly,

$$
Q_{y} h \in\left(Q, Q_{z}\right) .
$$

Thus, again applying Lemma 2.0.50 below, we find that $h \in\left(Q, Q_{z}\right)$, and hence

$$
H_{z} \in Q^{n-1}\left(Q, Q_{z}\right) \subseteq\left(Q^{n}\right)+(Q)_{z} .
$$

The above proof rested on the following basic result:

Lemma 2.0.50. If $f \in \mathbb{C}[x, y, z]$ satisfies

$$
Q_{x} f \in\left(Q, Q_{z}\right), \quad Q_{y} f \in\left(Q, Q_{z}\right)
$$

then it follows that

$$
f \in\left(Q, Q_{z}\right) .
$$

Proof. We claim that (2.0.51) implies that the ideal $(f) \subseteq \mathbb{C}[x, y, z] /\left(Q, Q_{z}\right)$ is a torsion module supported at the origin. Since (as we will recall), such torsion modules cannot be submodules of $\mathbb{C}[x, y, z] /\left(Q, Q_{z}\right)$, we will deduce that $f=0$.

To prove the claim, note that, since the singularity at the origin is isolated, at every closed point in $Z\left(Q, Q_{z}\right)$ other than the origin, either $Q_{x}$ or $Q_{y}$ must be nonvanishing. Hence, in every local ring other than at the origin, either $Q_{x}$ or $Q_{y}$ is a unit. Thus, $f$ is zero in every local ring other than the origin, i.e., $(f)$ is a torsion module supported at the origin.

Next, note that, since $Q$ is irreducible, $\mathbb{C}[x, y, z] /(Q)$ is a domain, and hence $Q, Q_{z}$ form a regular sequence in $\mathbb{C}[x, y, z]$. That is, we have a Koszul resolution of $\mathbb{C}[x, y, z] /\left(Q, Q_{z}\right)$ of length two. Since any torsion module $M$ supported at a point satisfies $\operatorname{Ext}^{i}(M, \mathbb{C}[x, y, z])=0$ for $i<3$, the long exact sequence of cohomology implies that $\operatorname{Ext}^{i}\left(M, \mathbb{C}[x, y, z] /\left(Q, Q_{z}\right)\right)=0$ for $i<1$. Thus, $\operatorname{Hom}\left((f), \mathbb{C}[x, y, z] /\left(Q, Q_{z}\right)\right)=0$. This implies that $f=0$, as desired.

To complete the proof of the proposition, first note that solutions to (2.0.29) such that $G \in \mathbb{C}[x, y] Q_{z}$, which form the subspace we wanted to quotient by, are exactly those for which the $F_{i} \in \mathbb{C}[x, y]$, and hence also $H \in \mathbb{C}[x, y]$. Therefore, to compute the Hilbert series of $V_{q}$, it remains, for each degree $|G|=m$, to find the dimension of the space of homogeneous $H$ of degree $|G|+q a-(d-c)$ such that $Q^{q-1} \mid H_{z}$, modulo the space of such $H$ which are polynomials in $x$ and $y$. In other words, we seek the dimension of the space of elements $H_{z}$ of degree $|G|+q a-d$ that are multiples of $Q^{q-1}$, modulo $(Q)_{z}$. By (2.0.45), this is equivalent to considering $\left(Q^{q-1}\right)=\left\{Q^{q-1} g \mid g \in \mathbb{C}[x, y, z]\right\}$ modulo $\left(Q^{q}\right)_{z}=\left\{Q^{q-1}\left(Q f_{z}+q Q_{z} f\right) \mid f \in\right.$ $\mathbb{C}[x, y, z]\}$. That is, writing $H_{z}=Q^{q-1} g$, our problem reduces to considering the space of polynomials $g$ of degree $|g|=|G|+q(a-d)$ modulo $\left\langle Q f_{z}+q Q_{z} f|| f|=| g \mid+(c-d)\right\rangle$. We conclude that the Hilbert series of $V_{q}$ is exactly (2.0.22).

\section{Proof of Theorem 1.1.13 when $X$ is not of type $A_{m-1}$}

We first consider the case where $X$ is not a singularity of type $A_{m-1}$. The main step left is to give the promised proof of Lemma 1.4.3.

Proof of Lemma 1.4.3. Let $\mathcal{G}_{X}=\exp \left(\mathfrak{g}_{X}\right)$ be the group of Poisson automorphisms of $\mathcal{O}_{\widehat{X}}$ generated by the flow of Hamiltonian vector fields $\xi_{f}$ for $f \in \mathcal{O}_{\widehat{X}}$ (so, $\left.\xi_{f}\right|_{g}=\{f, g\}$, identifying the tangent space at every point of $\mathcal{O}_{\widehat{X}}$ with $\mathcal{O}_{\widehat{X}}$ itself $)$. It is clear that $\mathcal{F}(X)^{\mathfrak{g}_{X}}=\mathcal{F}(X)^{\mathcal{G}_{X}}=$ $\mathbb{C}\left[(V \backslash\{0\}) \times \mathcal{O}_{\widehat{X}}^{0}\right]^{\mathcal{G}_{X}}$. 
The idea behind the proof of the lemma is to view the fibers of $Y$ as slices in $\alpha x+\beta y+\mathcal{O}_{\widehat{X}}^{0}$ to the orbits of the group $\mathcal{G}_{X}$, in the following sense.

For a fixed $(\alpha, \beta)$, note that $\alpha x+\beta y+\mathcal{O}_{\widehat{X}}^{0}$ is stable under $\mathcal{G}_{X}$ (since $\mathcal{O}_{\widehat{X}}^{0}$ contains all Poisson brackets). Hence, we have a map $\mathcal{O}_{\widehat{X}} / \mathcal{G}_{X} \rightarrow V$ with a canonical zero section $V:=\langle x, y\rangle \subseteq \mathcal{O}_{\widehat{X}} / \mathcal{G}_{X}$. Let $U^{\prime}$ be the pro-bundle over $V \backslash 0$ whose fiber at $\alpha x+\beta y$ is the tangent space to the fiber of the above map. In other words,

$$
U_{\alpha x+\beta y}^{\prime}=T_{\alpha x+\beta y}\left(\left(\alpha x+\beta y+\mathcal{O}_{\widehat{X}}^{0}\right) / \mathcal{G}_{X}\right) .
$$

The pro-bundle $U^{\prime}$ is evidently pulled back from a pro-bundle on $\mathbb{P}^{1}$. Call this $U$.

Claim 3.0.54. (i) The punctured plane $V \backslash\{0\} \subseteq \mathcal{O}_{\widehat{X}} / \mathcal{G}_{X}$ consists of smooth points.

(ii) We have a canonical isomorphism of pro-bundles

$$
U \cong Y \text {. }
$$

We will prove this claim below. For now, we assume it. Introduce the filtration on $\mathbb{C}\left[\mathcal{O}_{\widehat{X}} / \mathcal{G}_{X}\right]$ by powers of the ideal $I_{V}$ of functions vanishing on the plane $V$. By the claim, $V \backslash\{0\}$ consists of smooth points, and hence

$$
\operatorname{gr}_{I_{V}} \mathbb{C}\left[\mathcal{O}_{\widehat{X}} / \mathcal{G}_{X}\right]=\mathbb{C}\left[U^{\prime}\right] \cong \mathbb{C}\left[Y^{\prime}\right]
$$

where the latter denotes the global functions on the total space of the pro-bundle $Y^{\prime}$. The total space of $Y^{\prime}$ (a pro-bundle over $V \backslash\{0\}$ ), is the same as the total space of $E=Y \oplus \mathcal{O}(-1)_{a}$ (a pro-bundle over $\mathbb{P}^{1}$ ), and we deduce that

$$
\operatorname{gr}_{I_{V}} \mathbb{C}\left[\mathcal{O}_{\widehat{X}} / \mathcal{G}_{X}\right] \cong \mathbb{C}[E]
$$

By Theorem 1.4.17, $\mathbb{C}[E]$ is in fact a polynomial algebra on homogeneous generators, finitely many in each degree. Hence, the lemma follows from

Claim 3.0.58. Let $A$ be a graded commutative algebra $A$ with a descending graded filtration $A=F_{0} A \supseteq F_{1} A \supseteq \cdots$ with $\cap_{i} F_{i} A=0$ such that gr $A \cong$ Sym $W$, where $W$ is a bigraded vector space which is finite-dimensional in each bidegree. Then,

$$
A=\operatorname{Sym} \widetilde{W} \cong \operatorname{gr} A,
$$

for any graded lifting $\widetilde{W}$ of $W$ to $A$.

Proof. We have a canonical morphism of algebras, $\iota:$ Sym $\widetilde{W} \rightarrow A$, which becomes an isomorphism when we take associated graded. Hence, it must be a monomorphism. To prove surjectivity, fix a degree $n \geq 0$. The surjectivity of Sym $W \rightarrow \operatorname{gr} A$ says that

$$
\iota(\operatorname{Sym} \widetilde{W})_{n}+\left(F_{i+1} A\right)_{n} \supseteq\left(F_{i} A\right)_{n}, \forall i .
$$

Since $\cap_{i} F_{i} A=0$ and each $\left(F_{i} A\right)_{n}$ is finite-dimensional, there must exist $j \geq 0$ such that $\left(F_{j+1} A\right)_{n}=0$. We deduce from (3.0.60) that $\iota(\operatorname{Sym} \widetilde{W})_{n} \supseteq\left(F_{j} A\right)_{n}$, and applying (3.0.60) $j$ more times, we deduce that $\iota(\operatorname{Sym} \widetilde{W})_{n} \supseteq\left(F_{0} A\right)_{n}=A_{n}$.

We apply this for $A=\mathbb{C}\left[\mathcal{O}_{\widehat{X}} / \mathcal{G}_{X}\right]$, with the filtration by powers of $I_{V}$, and $A_{0}=\mathbb{C}[E]$.

Proof of Claim 3.0.54. Since $\mathcal{O}_{\hat{X}}$ is a $\mathcal{G}_{X}$-representation, for any element $f \in \mathcal{O}_{\hat{X}}$, we have

$$
T_{\alpha x+\beta y+\hbar f}\left(\mathcal{O}_{\hat{X}} / \mathcal{G}_{X}\right)=\left\{\alpha x+\beta y+\hbar f, \mathcal{O}_{\hat{X}}\right\}
$$


It suffices to show that the RHS is saturated as a $\mathbb{C}[[\hbar]]$-module, i.e., if

$$
\{\alpha x+\beta y+\hbar f, g\} \in \hbar \mathcal{O}_{\hat{X}}[[\hbar]],
$$

then

$$
g \in Z_{\alpha x+\beta y+\hbar f}+\hbar \mathcal{O}_{\hat{X}}[[\hbar]],
$$

where $Z_{\alpha x+\beta y+\hbar f}$ is the Poisson centralizer. Since $Z_{\alpha x+\beta y}=\mathbb{C}[[\alpha x+\beta y]]$, (3.0.62) can only hold if $g \in \mathbb{C}[[\alpha x+\beta y]]+\hbar \mathcal{O}_{\hat{X}}[[\hbar]]$. Then, using that $\mathbb{C}[[\alpha x+\beta y+\hbar f]] \subseteq Z_{\alpha x+\beta y+\hbar f}$ (in fact, this is an equality), we see that (3.0.63) holds.

Now, Theorem 1.1 .13 (in the non-type $A$ case) follows from Theorem 1.4.17, since we have identified the regular invariant functions with the regular functions on the total space of $E=$ $Y \oplus \mathcal{O}(-1)_{a}$. That is, we take the global sections of Sym $E^{*}=\operatorname{Sym} Y^{*} \otimes \bigoplus_{m \geq 0}\left(\mathcal{O}(1)_{-a}\right)^{\otimes m}$. If we decompose $Y=\prod_{i} \mathcal{O}\left(n_{i}\right)_{m_{i}}$, then the desired regular functions form a polynomial algebra on the generators $f_{i} \in \Gamma\left(\mathbb{P}^{1}, \mathcal{O}\left(-n_{i}\right)_{-m_{i}} \otimes\left(\mathcal{O}(1)_{-a}\right)^{\otimes n_{i}}\right) \backslash\{0\}$, of weight $-m_{i}-n_{i} a$ and polynomial degree $n_{i}+1$ (these vector spaces are one-dimensional, so any nonzero $f_{i}$ will work), together with the generators $\alpha, \beta$, which are the sections of $\mathcal{O}(1)_{-a}$. Letting $L$ again denote the span of these generators, we see from (1.4.18) that

$$
\begin{aligned}
h\left(L ; t^{-1}\right)=\sum_{i=0}^{\infty} s^{i+1} t^{i a} t^{i(d-a)} \frac{\left(1-t^{d-a}\right)\left(1-t^{d-b}\right)\left(1-t^{d-c}\right)}{\left(1-t^{a}\right)\left(1-t^{b}\right)\left(1-t^{c}\right)} \\
\quad=\frac{s\left(1-t^{d-a}\right)\left(1-t^{d-b}\right)\left(1-t^{d-c}\right)}{\left(1-t^{a}\right)\left(1-t^{b}\right)\left(1-t^{c}\right)\left(1-t^{d} s\right)}=\frac{h\left(J_{Q} ; t\right) s}{1-t^{d} s},
\end{aligned}
$$

using the well-known formula for $h\left(J_{Q} ; t\right)$ (which says that $\left(Q_{x}, Q_{y}, Q_{z}\right)$ form a regular sequence in $\mathbb{C}[x, y, z])$. This completes the proof of Theorem 1.1.13 in the non- $A_{m-1}$ case.

\section{Proof of Theorem 1.1.13 in the $A_{m-1}$ CASE}

This case involves the Poisson algebra $C[X]=\mathbb{C}[x, y, z] /(Q)$ for $Q=x^{m}+y^{2}+z^{2}$. It will be convenient to present this slightly differently, as $\mathcal{O}_{X}=\mathbb{C}\left[\mathrm{x}^{m}, \mathrm{xy}, \mathrm{y}^{m}\right] \subset \mathbb{C}[\mathrm{x}, \mathrm{y}]$, with the usual Poisson bracket $\{\mathrm{x}, \mathrm{y}\}=1$ (this is the natural presentation from the point of view $X=\mathbb{C}^{2} /(\mathbb{Z} / m)$ ), and $|\mathrm{x}|=|\mathrm{y}|=1$. As before, define $\mathcal{F}(X)$ as in (1.4.2). We once again have that $\mathbb{C}\left[\mathcal{O}_{X}\right]^{\mathfrak{g}_{X}}$ is (a completion of $) \mathcal{F}(X)^{\mathfrak{g}_{X}} \cong \mathcal{F}(X)^{\mathcal{G}_{X}}$, where $\mathcal{G}_{X}=\exp \left(\mathfrak{g}_{X}\right)$ is the group of Poisson automorphisms of $\mathcal{O}_{\widehat{X}}=\mathbb{C}\left[\left[\mathrm{x}^{m}, \mathrm{xy}, \mathrm{y}^{m}\right]\right]$ obtained from the flow of Hamiltonian vector fields. Also, since it will turn out that $\mathcal{F}(X)^{\mathcal{G}_{X}}$ is finite-dimensional in each degree $\mathbb{A}^{4}$ in fact $\mathbb{C}\left[\mathcal{O}_{X}\right]^{\mathfrak{g}_{X}} \cong \mathcal{F}(X)^{\mathcal{G}_{X}}$ as graded vector spaces. Now, Theorem 1.1.13 will follow from the following replacement for Theorem 1.4.17;

Proposition 4.0.65. The following set is a slice to the $\mathcal{G}_{X}$-orbits in $\mathbb{C}\left[\left[\mathrm{x}^{m}, \mathrm{xy}, \mathrm{y}^{m}\right]\right]$ with nonzero coefficient of $\mathrm{y}^{m}$ or of $\mathrm{xy}$ :

$$
\mathrm{y}^{m}+\mathbb{C}\left[\left[\mathrm{x}^{m}\right]\right]\left\langle 1, \mathrm{xy},(\mathrm{xy})^{2}, \ldots,(\mathrm{xy})^{m-2}\right\rangle .
$$

\footnotetext{
${ }^{4}$ This is also a consequence of the known fact that $H P_{0}\left(\mathbb{C}\left[\mathbb{C}^{2 n}\right]^{(\mathbb{Z} / m)^{n} \rtimes S_{n}}\right)$ is finite-dimensional, a special case of the result of the appendix to $\mathrm{BEG04}$, that $H P_{0}\left(\mathbb{C}\left[\mathbb{C}^{2 n}\right]^{G}\right)$ is finite-dimensional for all finite $G<\mathrm{Sp}_{2 n}$.
} 
Proof. If the coefficient of $\mathrm{y}^{m}$ is zero but not the coefficient of xy, we can apply $e^{\left.\xi_{(\mathrm{y} m)}\right)}$ to make the coefficient of $\mathrm{y}^{m}$ nonzero. Once we have a nonzero coefficient of $\mathrm{y}^{m}$, by applying rescalings $\mathrm{y}^{m} \mapsto \gamma \mathrm{y}^{m}, \mathrm{x}^{m} \mapsto \gamma^{-1} \mathrm{x}^{m}$, we can make the coefficient of $\mathrm{y}^{m}$ one. Next, we use the lexicographical ordering $\prec$ on monomials $\mathbb{C}\left[\left[\mathrm{x}^{m}, \mathrm{xy}, \mathrm{y}^{m}\right]\right]$, where $\mathrm{x}^{a} \mathrm{y}^{b} \prec \mathrm{x}^{a^{\prime}} \mathrm{y}^{b^{\prime}}$ if either $a<a^{\prime}$ or $a=a^{\prime}$ and $b<b^{\prime}$. Note that, for $a \neq 0$ and $f \in \mathbb{C}\left[\left[\mathrm{x}^{m}, \mathrm{xy}, \mathrm{y}^{m}\right]\right]$ a power series with zero coefficient of $\mathrm{y}^{m}$,

$$
\left\{\mathrm{x}^{a} \mathrm{y}^{b}, \mathrm{y}^{m}+f\right\}=\left\{\mathrm{x}^{a} \mathrm{y}^{b}, \mathrm{y}^{m}\right\}+\ldots, \quad e^{\xi_{\left(\mathrm{x}^{a} \mathrm{y}^{b}\right)}}\left(\mathrm{y}^{m}+f\right)=\left\{\mathrm{x}^{a} \mathrm{y}^{b}, \mathrm{y}^{m}\right\}+\ldots,
$$

where ... denotes higher-order terms with respect to $\prec$. Hence, by applying elements $e^{\xi_{\left(x^{a} y^{b}\right)}}$, we can kill off all monomials which appear in $\left\{\mathrm{y}^{m}, \mathbb{C}\left[\left[\mathrm{x}^{m}, \mathrm{xy}, \mathrm{y}^{m}\right]\right]\right\}$, which is a complement to $\mathbb{C}\left[\left[\mathrm{x}^{m}\right]\right]\left\langle 1, \mathrm{xy},(\mathrm{xy})^{2}, \ldots,(\mathrm{xy})^{m-2}\right\rangle$. Hence, any orbit with nonzero coefficient of either $\mathrm{xy}$ or $\mathrm{y}^{m}$ contains a point of the form (4.0.66).

It remains to prove that this point is unique. In other words, if $g \in \mathbb{C}\left[\left[\mathrm{x}^{m}, \mathrm{xy}, \mathrm{y}^{m}\right]\right]$ satisfies

$$
e^{\xi_{g}}\left(\mathrm{y}^{m}+f\right)=\mathrm{y}^{m}+f^{\prime}, \quad f, f^{\prime} \in \mathbb{C}\left[\left[\mathrm{x}^{m}\right]\right]\left\langle 1, \mathrm{xy},(\mathrm{xy})^{2}, \ldots,(\mathrm{xy})^{m-2}\right\rangle,
$$

then $f=f^{\prime}$. It suffices to show that, if $f \neq f^{\prime}$, then the lowest-order term in $f-f^{\prime}$ with respect to $\prec$ lies in $\left\{\mathrm{y}^{m}, \mathbb{C}\left[\left[\mathrm{x}^{m}, \mathrm{xy}, \mathrm{y}^{m}\right]\right]\right\}$ (since this is impossible).

We assume that $f$ and $f^{\prime}$ have no constant term. Note that, for any power series $R\left(\mathrm{y}^{m}+f\right)$ in $\mathrm{y}^{m}+f$, the operator $e^{\xi_{R\left(\mathrm{y}^{m}+f\right)}}$ fixes $\mathrm{y}^{m}+f$. Using the Campbell-Baker-Hausdorff formula, we can replace $g$ by an element $g^{\prime}$ such that $e^{\xi_{g^{\prime}}}=e^{\xi_{g}} e^{\xi_{R\left(y^{m}+f\right)}}$. Hence, inductively on $\prec$, we may assume that the coefficient in $g$ of every monomial $\mathrm{y}^{\mathrm{km}}$ is zero. In this case, the lowest-order term in $f-f^{\prime}$ with respect to $\prec$ appears in $\left\{\mathrm{y}^{m}, g\right\}$.

Corollary 4.0.69. The invariant regular functions $\mathcal{F}(X)^{\mathcal{G}_{X}}$ restrict isomorphically to the regular functions on the slice (4.0.66).

Proof. By the proposition, restriction to the slice (4.0.66) identifies regular $\mathcal{G}_{X}$-invariant functions on the subvariety $U$ of $\mathbb{C}\left[\left[\mathrm{x}^{m}, \mathrm{xy}, \mathrm{y}^{m}\right]\right]$ consisting of power series whose coefficient of $\mathrm{y}^{m}$ or xy is nonzero with regular functions on (4.0.66). Since $U$ is the complement of an affine subspace of codimension two, all regular (invariant) functions on $U$ extend to regular (invariant) functions on all of $\mathbb{C}\left[\left[\mathrm{x}^{m}, \mathrm{xy}, \mathrm{y}^{m}\right]\right]$.

It remains to compute the algebra of regular functions on (4.0.66). This is a polynomial algebra generated by the coordinate functions $w_{\mathrm{x}^{r} \mathrm{y}^{s}}$ of the slice, by which we mean that the point with coordinates $\left(w_{\mathrm{x}^{r} \mathrm{y}^{s}}\right)$ in the slice is

$$
\mathrm{y}^{m}+\sum_{r, s} w_{\mathrm{x}^{r} \mathrm{y}^{s}} \mathrm{x}^{r} \mathrm{y}^{s}
$$

These do not necessarily have degree one as polynomial functions on $\mathcal{O}_{\hat{X}}$. To determine the degree we may consider the value of the slice coordinate $w_{\mathrm{x}^{r} \mathrm{y}^{s}}$ on $\gamma$ times (4.0.70) for arbitrary $\gamma \in \mathbb{C}$. We can compute this by applying the element of $\mathcal{G}_{X}$ which rescales by $\mathrm{y}^{m} \mapsto \gamma^{-1} \mathrm{y}^{m}$ and $\mathrm{x}^{m} \mapsto \gamma \mathrm{x}^{m}$. We deduce that the degree of $w_{\mathrm{x}^{r} \mathrm{y}^{s}}$ is $\frac{r-s}{m}+1$. This yields exactly (1.1.14), proving Theorem 1.1.13 in the $A_{m-1}$ case. 


\section{Proof of Theorems 1.2.1 and 1.2.2 and Corollary 1.2 .3}

To prove this, we first need to recall the structure of the zeroth Hochschild homology of symmetric products of algebras. Form the coalgebra

$$
\mathcal{H}(A):=\bigoplus_{n \geq 0} \mathrm{HH}_{0}\left(\operatorname{Sym}^{n} A\right),
$$

where the comultiplication map is given by the symmetrization maps

$$
\operatorname{Sym}^{p} A \rightarrow \bigoplus_{m+n=p} \operatorname{Sym}^{m} A \otimes \operatorname{Sym}^{n} A .
$$

(When $\mathrm{HH}_{0}(A)$ is finite-dimensional, this is dual to the symmetrization maps that we considered earlier.) We then have the following result:

Theorem 5.0.73. [EO06, Corollary 3.3] Let $A$ be an infinite-dimensional simple algebra over $\mathbb{C}$ with trivial center. Then, the algebra $\mathcal{H}(A)$ is a polynomial coalgebra,

$$
\mathcal{H}(A) \cong \operatorname{Sym}\left(\mathrm{HH}_{0}(A)[t]\right),
$$

where the isomorphism is the unique graded coalgebra map (with $\mathrm{HH}_{0}(A)$ and $t$ both in degree one) such that the composition with the projection to $t^{n-1} \mathrm{HH}_{0}(A)$,

$$
\mathrm{HH}_{0}\left(\operatorname{Sym}^{n} A\right) \rightarrow \operatorname{Sym}\left(\mathrm{HH}_{0}(A)[t]\right) \rightarrow t^{n-1} \mathrm{HH}_{0}(A),
$$

has the form $\left[a_{1} \& \cdots \& a_{n}\right] \mapsto \frac{1}{n !} \sum_{\sigma \in S_{n}} t^{n-1}\left[a_{\sigma(1)} \cdots a_{\sigma(n)}\right]$.

We note that the above theorem is not stated in quite this way in [EO06], but rather in the equivalent formulation that

$$
\mathrm{HH}_{0}\left(\operatorname{Sym}^{n} A\right) \cong \bigoplus_{\nu \in \mathcal{P}_{n}} \bigotimes_{i \geq 1} \operatorname{Sym}^{\nu_{i}} \mathrm{HH}_{0}(A),
$$

where $\mathcal{P}_{n}$ is the set of partitions of $n$, and $\nu_{i}$ denotes the number of cells of $\nu$ of size $i$.

We have the following immediate corollary:

Corollary 5.0.77. In the situation of Theorem 5.0.73, if $\mathrm{HH}_{0}(A)$ is finite-dimensional, then the commutative algebra $\bigoplus_{n>0} \mathrm{HH}_{0}\left(\mathrm{Sym}^{n} A\right)^{*}$ is freely generated by the vector spaces $\left\langle\left[f^{\& n}\right] \mapsto T\left(\left[f^{n}\right]\right)\right\rangle_{T \in \mathrm{HH}_{0}(A)^{*}} \subseteq \mathrm{HH}_{0}\left(\overline{S y m}^{n} A\right)^{*}$.

Proof of Theorem 1.2.1. The algebra $\mathrm{Weyl}_{2 n}^{H}$ is well-known to be simple for all finite groups $H<\mathrm{Sp}_{2 n}$. Indeed, since $\mathrm{Weyl}_{2 n}$ is simple, so is the smash-product algebra $\mathrm{Weyl}_{2 n} \rtimes H$, and this is therefore Morita equivalent to $\mathrm{Weyl}_{2 n}^{H}$. Obviously, Weyl ${ }_{2 n}^{H}$ is also infinite-dimensional. Since, for finite $G<\mathrm{Sp}_{2}$ and $X=\mathbb{C}^{2} / G$, it is known that $\mathrm{HH}_{0}\left(\mathrm{Weyl}_{2}^{G}\right) \cong \operatorname{HP}_{0}\left(\mathcal{O}_{X}\right)$, (e.g. by comparing [AFLS00] for the former with the formulas mentioned in the introduction for the latter), the theorem follows from Theorem 5.0.73 and the main Theorem 1.1.13.

Proof of Theorem 1.2.2. Although the deformation quantization of $\mathcal{O}_{X}$ is not, in general, simple, we may deform $X$ to $Z(Q-\lambda)$, which is symplectic for $\lambda \neq 0$. Let $A_{\lambda}:=$ $\mathbb{C}[x, y, z] /(Q-\lambda)$, and let $A_{\lambda, \hbar}$ be its deformation quantization; the algebra $A_{\lambda, \hbar}\left[\hbar^{-1}\right]$ is simple. By results of Nest-Tsygan [NT95], we have $\mathrm{HH}_{0}\left(A_{\lambda, \hbar}\left[\hbar^{-1}\right]\right) \cong \operatorname{HP}_{0}\left(A_{\lambda}\right)((\hbar))$ for $\lambda \neq 0$, i.e., the Brylinski spectral sequence degenerates. Moreover, the Betti numbers of $A_{\lambda}$ are 1,0 , and $\mu_{Q}$, where $\mu_{Q}=\operatorname{dim} \mathbb{C}[x, y, z] /\left(Q_{x}, Q_{y}, Q_{z}\right)$ is the Milnor number of $X$. Hence, $\operatorname{dim}_{\mathbb{C}((\hbar))} \mathrm{HH}_{0}\left(A_{\lambda, \hbar}\left[\hbar^{-1}\right]\right)=\operatorname{dim} \mathrm{HP}_{0}\left(A_{0}\right)$. By Theorem 5.0.73 and Theorem 1.1.13, 
we deduce that $\mathrm{HH}_{0}\left(\operatorname{Sym} A_{\lambda, \hbar}\left[\hbar^{-1}\right]\right) \cong \mathrm{HP}_{0}\left(\operatorname{Sym} A_{0}\right)((\hbar))$ as graded algebras (with degree $n$ corresponding to $\mathrm{Sym}^{n}$, so not looking at the grading on $A_{0}$ yet). However, as $\lambda \rightarrow 0$, $\mathrm{HH}_{0}\left(\operatorname{Sym}^{n} A_{\lambda, \hbar}\left[\hbar^{-1}\right]\right)$ can only increase in dimension over $\mathbb{C}((\hbar))$, but the Brylinski spectral sequence shows that $\operatorname{dim}_{\mathbb{C}((\hbar))} \mathrm{HH}_{0}\left(\operatorname{Sym}^{n} A_{0, \hbar}\left[\hbar^{-1}\right]\right) \leq \operatorname{dim} \mathrm{HP}_{0}\left(\operatorname{Sym}^{n} A_{0}\right)$ for all $n$. Hence, the dimensions are equal, and the Brylinski spectral sequence degenerates.

Proof of Corollary 1.2.3. In a formal punctured neighborhood of $\gamma=0$, we see from the above that the zeroth Hochschild homology is constant and as above. Hence, by a standard argument, the same is true when $\hbar$ is replaced by actual values of $\gamma$ that do not obey a countable number of polynomial equations, i.e., for all but countably many $\gamma$, and the zeroth Hochschild homology is as above.

Remark 5.0.78. We deduce from the above that, for the elliptic algebras $A_{\gamma}$, the two sides of (5.0.74) are abstractly isomorphic as bigraded algebras (Corollary 1.2.3). However, the map defined in Theorem 5.0.73 is not an isomorphism: for example, consider $T \in \mathrm{HH}_{0}(A)^{*}$ which takes the degree-zero coefficient of an element of $A /[A, A]$. Then, $T\left(\left[a^{2}\right]\right)=T([a])^{2}$, so the map is not an isomorphism (cf. Corollary 5.0.77). Moreover, the map of Theorem 5.0.73 is not even a bigraded map: while it preserves degree (and is thus a graded map), it does not preserve weight: while $\left|t^{n-1} T\right|=-(n-1) d+|T|$ on the RHS for $T \in \mathrm{HH}_{0}\left(A_{\gamma}\right)^{*}$, the element $\left[f^{\& n}\right] \mapsto T\left(\left[f^{n}\right]\right)$ has degree $|T|$ on the LHS. So the fact that an isomorphism between the two sides of (5.0.74) exists is subtle and there may not be a canonical one.

\section{ACKNOWLEDGEMENTS}

We thank Qingchun Ren for writing computer programs which allowed us to compute $\mathrm{HP}_{0}$ for certain surfaces in low degrees (as part of an MIT UROP with the authors). We are grateful to J. Alev for useful discussions. The first author is partially supported by NSF grant DMS-0504847. The second author is a five-year fellow of the American Institute of Mathematics.

\section{REFERENCES}

[AF] J. Alev and L. Foissy, Le groupe des traces de Poisson de la varieté quotient $h+h^{*} / W$ en rang 2, arXiv:math/0603142.

[AFLS00] J. Alev, M. A. Farinati, T. Lambre, and A. L. Solotar, Homologie des invariants d'une algèbre de Weyl sous l'action d'un groupe fini, J. Algebra 232 (2000), 564-577.

[AL98] J. Alev and T. Lambre, Comparaison de l'homologie de Hochschild et de l'homologie de Poisson pour une déformation des surfaces de Klein, Algebra and operator theory (Tashkent, 1997) (Dordrecht), Kluwer Acad. Publ., 1998, pp. 25-38.

[ATdB90] M. Artin, J. Tate, and M. Van den Bergh, Some algebras associated to automorphisms of elliptic curves, Grothendieck Festschrift, Vol. I, Progr. Math., vol. 86, Birkhäuser Boston, 1990, pp. 33-85.

[BEG04] Y. Berest, P. Etingof, and V. Ginzburg, Morita equivalence of Cherednik algebras, J. Reine Angew. Math. 568 (2004), 81-98, arXiv:math/0207295.

[But08] F. Butin, Poisson homology in degree 0 for some rings of symplectic invariants, arXiv:0809.4983, 2008.

[EG07] P. Etingof and V. Ginzburg, Noncommutative del Pezzo surfaces and Calabi-Yau algebras, arXiv:0709.3593v2, 2007.

[EO06] P. Etingof and A. Oblomkov, Quantization, orbifold cohomology, and Cherednik algebras, Jack, Hall-Littlewood and Macdonald polynomials (Providence, RI), Contemp. Math., vol. 417, Amer. Math. Soc., 2006, arXiv:math/0311005, pp. 171-182.

[NT95] R. Nest and B. Tsygan, Algebraic index theorem, Comm. Math. Phys. 172 (1995), no. 2, 223-262. 
[Sai87] K. Saito, Regular system of weights and associated singularities, Complex analytic singularities (Amsterdam), Adv. Stud. Pure Math., vol. 8, North-Holland, 1987, pp. 479-526.

[Ste97] D. R. Stephenson, Algebras associated to elliptic curves, Trans. Amer. Math. Soc. 349 (1997), 2317-2340.

[VdB01] M. Van den Bergh, Blowing up of non-commutative smooth surfaces, vol. 154, Mem. Amer. Math. Soc., no. 734, Amer. Math. Soc., Providence, RI, 2001. 